\title{
On a Sequence of Points of Interest for Numerical Quadrature
}

\section{Seymour Haber}

\author{
Institute for Basic Standards, National Bureau of Standards, Washington, D.C.
}

(February 15, 1966)

The distribution of 2 sequences of points, originally discussed by van der Corput and by K. F. Roth, is studied in detail. The results obtained disprove a conjecture of $\mathrm{J}$. H. Halton and suggest a conjecture on the improvement of a theorem of Roth.

Key Words: Uniform distribution, equidistribution, distribution functions, diophantine approximation, quadrature, Monte Carlo methods, multiple quadrature, integration, numerical analysis, distribution of points.

1. If $x_{1}, x_{2}, \ldots, x_{N}$ are points in the unit $S$-dimensional cube $G_{S}$, we may approximate to an integral over $G_{S}$ by the average of the values of the integrand at these points. Writing

$$
\int_{0}^{1} \ldots \int_{0}^{1} f\left(x^{(1)}, x^{(2)}, \ldots, x^{(S)}\right) d x^{(1)} \ldots d x^{(S)}=\frac{1}{N} \sum_{i=1}^{N} f\left(x_{i}^{(1)}, \ldots, x_{i}^{(S)}\right)+R
$$

(where $x_{i}^{(1)}, x_{i}^{(2)} \ldots, x_{i}^{(S)}$ are the $S$ coordinates of the point $x_{i}$ ), we call $R$ the "error" of the quadrature formula (1). $R$ depends on the integrand $f$ and on the set of points $x_{1}, \ldots, x_{N}$; for $R$ to be relatively small for some wide class of functions the set of points should be, in some sense, well distributed in the cube $G_{S}$. An appropriate sense of "well distributed" is that, for a region $A$ in $G_{S}$, the number of points of the set which lie in $A$ should be approximately $N$ times the volume of $A$. Restricting attention to regions which are intervals with one vertex at the origin-i.e., which are of the form

$$
\left\{x=\left(x^{(1)}, \ldots, x^{(S)}\right) \mid 0 \leqslant x^{(i)}<x_{0}^{(i)}, i=1,2, \ldots, S\right\}
$$

for some given $x_{0}=\left(x_{0}^{(1)}, \ldots, x_{0}^{(S)}\right) \epsilon G_{S}$ - we shall let $S_{N}\left(x_{0}^{(1)}, \ldots, x_{0}^{(S)}\right)$ denote the number of the points $x_{1}, \ldots x_{N}$ which lie in such an interval, and consider the quantity $\mid S_{N}\left(x_{0}^{(1)}, \ldots, x_{0}^{(S)}\right)$ $-N x_{0}^{(1)} x_{0}^{(2)}, \ldots x_{0}^{(S)} \mid$.

Let

$$
D_{N}^{*}=D_{N}^{*}\left(x_{1}, \ldots, x_{N}\right)=\sup \left|S_{N}\left(x_{0}^{(1)}, \ldots, x_{0}^{(S)}\right)-N x_{0}^{(1)}, \ldots, x_{0}^{(S)}\right| .
$$

$D_{N}^{*}$ is very nearly the "discrepancy" (see, e.g., [1]) ${ }^{1}$ of the set of $N$ points. E. Hlawka [2] has shown that if the integrand $f$ in (1) is a function of bounded variation and $V(f)$ is its total variation, we have the bound

$$
|R| \leqslant V(f) \frac{D_{N}^{*}}{N}
$$

\footnotetext{
${ }^{1}$ Figures in brackets indicate the literature references at the end of this paper.
} 
Since it is very likely true that all continuous and bounded functions that one ever attempts to integrate numerically are of bounded variation, it is a matter of considerable interest to find sets of points for which $D_{N}^{*}$ is as small as possible.

It is easy to see that $D_{N}^{*} \geqslant \frac{1}{2}$. For if $\left|S_{N}\left(x_{1}^{(1)}, 1, \ldots, 1\right)-N x_{1}^{(1)}\right|=a<\frac{1}{2}$, then for any $\epsilon>0$ $\left|S_{N}\left(x_{1}^{(1)}+\epsilon, 1, \ldots, 1\right)-N\left(x_{1}^{(1)}+\epsilon\right)\right|>1-a-N \epsilon$ and so is $>\frac{1}{2}$ if $\epsilon$ is sufficiently small. In one dimension, the sequence of points $x_{i}=\frac{2 i-1}{2 N}, i=1,2, \ldots, N$, has $D_{N}^{*}=\frac{1}{2}$ for every $N$. In two or more dimensions the situation is entirely different; K. F. Roth [3] has shown that for $S>1$ dimensions there is a positive number $C(S)$ such that

$$
D_{N}^{*}>C(S)(\log N)^{\frac{S-1}{2}}
$$

for any set of $N$ points. It is not known that this result is best possible; there is some reason to guess that the exponent $\frac{S-1}{2}$ may be replaceable by $S-1$. In his proof Roth introduced another measure of unevenness of distribution, which we shall denote " $E_{N}$ ". It is defined by

$$
E_{N}=E_{N}\left(x_{1}, \ldots, x_{N}\right)=\left(\int_{0}^{1} \ldots \int_{0}^{1}\left|S_{n}\left(x_{1}^{(1)} \ldots, x^{(S)}\right)-N x^{(1)}, \ldots, x^{(S)}\right|^{2} d x^{(1)} \ldots d x^{(S)}\right)^{1 / 2}
$$

This $L^{2}$ norm is sometimes easier to handle than the maximum norm $D_{N}^{*}$, and Roth in fact proved (4) with $E_{N}$ in place of $D_{N}^{*}$.

In 1935 van der Corput [4] raised the question of whether there exists an infinite sequence $x_{1}, x_{2}, \ldots$ of points in the inverval $[0,1]$ such that $D_{N}^{*}\left(x_{1}, x_{2}, \ldots, x_{N}\right)$ is bounded in $N$. This was answered in the negative by Mrs. van Aardenne-Ehrenfest [5] in 1945. Roth, in [3], showed that this problem is equivalent to the problem of estimating a lower bound for $D_{N}^{*}$ for fixed $N$ in two dimensions. More generally, Roth showed the existence of a constant $C$ such that: For any $N$ and $S$, if there is a set of points $x_{1}, \ldots, x_{N}$ in $G_{S}$ such that $D_{N}^{*}\left(x_{1}, \ldots, x_{N}\right)=A$, there is a sequence $x_{1}^{\prime}, \ldots, x_{N}^{\prime}$ in $G_{S-1}$ such that $\max _{n \leq N} D_{n}^{*}\left(x_{1}^{\prime}, \ldots, x_{n}^{\prime}\right)<C A$; and conversely. It follows that for any infinite sequence $x_{1}, x_{2}, \ldots$ in $[0,1], D_{N}^{*}\left(x_{1}, \ldots, x_{N}\right) \geqslant C^{\prime}(\log N)^{1 / 2}$ for infinitely many values of $N$ (where $C^{\prime}$ is some positive constant). Van der Corput found a sequence for which he showed that $D_{N}^{*} \leqslant \log _{2} N+1$ (here and below " $\log _{2}$ " denotes the logarithm to the base two); it is constructed as follows: for each integer $n \geqslant 0$, write $n$ as $2^{h_{1}}+2^{h_{2}}+\ldots+2^{h_{k}}$ with $h_{1}<h_{2}<\ldots .<h_{k}$; then $x_{n}=\frac{1}{2}\left(2^{-h_{1}}+2^{-h_{2}}+\ldots+2^{-h_{k}}\right)$. (We may picture this process as writing $n$ as a numeral to the base 2 and reflecting this numeral in the "decimal point." Thus, in binary notation, $x_{0}=0, x_{1}=0.1$, $x_{2}=0.01, x_{3}=0.11, x_{4}=0.001$, etc.) Roth pointed out that then for each $N$, the sequence $\left(x_{1}, \frac{1}{N}\right)$, $\left(x_{2}, \frac{2}{N}\right), \ldots,\left(x_{N}, 1\right)$ in $G_{2}$ has the property that $D_{N}^{*} \leqslant \log _{2} N+2$. We shall refer to the van der Corput sequence as " $\mathscr{S}$ " and to the Roth sequence, for any $N$, as " $\mathscr{S}$ ".

J. M. Hammersley [6] and J. H. Halton [7], interested in the application of evenly distributed sequences to high-dimensional numerical integration, suggested higher-dimensional analogs of these sequences. Defining $\varphi_{k}(n)$, for any integers $n \geqslant 0$ and $k>1$, as the number produced by reflecting the representation of $n$ to the base $k$ in the "decimal point" (more formally, if $n=a_{1} k^{h_{1}}$

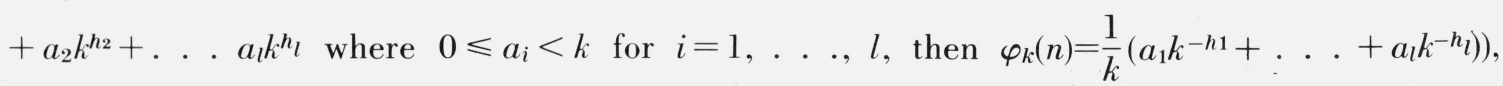
Halton suggested the $s$-dimensional sequence defined by

$$
x_{n}=\left(\varphi_{2}(n), \varphi_{3}(n), \varphi_{5}(n), \ldots, \varphi_{P}(n)\right), n=0,1,2, \ldots
$$


(where $P_{s}$ is the $S$ th prime) and Hammersley suggested

$$
x_{n}=\left(\frac{n}{N}, \varphi_{2}(n), \varphi_{3}(n), \ldots, \varphi_{P_{S-1}}(n)\right), n=1,2, \ldots, N
$$

Halton proved that for the sequence $(6), D_{N}^{*}$ and $E_{N}$ are $\leqslant C(\log N)^{K}$ and for $(7)$ they are $\leqslant C^{\prime}(\log$ $N)^{K-1}$, for some constants $C$ and $C^{\prime}$. He further conjectured that the exponents $K$ and $K-1$ could be replaced by $K / 2$ and $(K-1) / 2$ respectively.

2. In this paper, we shall analyze the distribution of the sequences $\mathscr{S}$ and $\mathscr{S}^{\prime}$ in $\operatorname{detail}$. Our results are as follows:

THEOREM 1. For the sequence $\mathscr{S}$,

$$
D_{N}^{*} \leqslant \frac{1}{3} \log _{2}(N)+0(1)
$$

and the constant $1 / 3$ is best possible-that is, with any smaller constant (8) would not always be true.

THEOREм 2. For the sequence $\mathscr{S}$,

$$
E_{N} \leqslant \frac{1}{6} \log _{2} N+0(1)
$$

and the constant $1 / 6$ is best possible.

THeOREM 3. For the sequence $\mathscr{S}^{\prime}$,

$$
D_{N}^{*}=\frac{1}{3} \log _{2} N+0(1)
$$

THEOREM 4. For the sequence $\mathscr{S}^{\prime}$,

$$
E_{N}=\frac{1}{8} \log _{2} N+0(1)
$$

From these theorems it follows that Halton's conjecture is incorrect for the lowest-dimensional cases of his and Hammersley's sequences; it is very likely also to be incorrect in all dimensions.

3. Proofs of the Theorems:

We shall first make some notational conventions.

If a letter is used to represent a nonnegative integer, the same letter may be used to represent the finite string of zeros and ones which is the binary representation of that integer.

If $\alpha$ is a finite string of zeros and ones, we shall enumerate its digits from right to left, calling the rightmost one the "zeroth digit," and write " $\alpha=\alpha_{k} \ldots \alpha_{1} \alpha_{0}$." The number of digits in $\alpha$ will be denoted " $\|\alpha\|$ "; thus $\left\|\alpha_{k} \alpha_{k-1} \ldots \alpha_{0}\right\|=k+1$. For $0 \leqslant i \leqslant k$, " $\alpha^{(i) " ~}$ will denote the string $\alpha_{i} \alpha_{i-1} \ldots \alpha_{1} \alpha_{0}$.

For a string $\alpha=\alpha_{k} \ldots \alpha_{0}$, ". $\alpha$ " will denote the number $\frac{\alpha_{k}}{2}+\frac{\alpha_{k-1}}{4}+\ldots+\frac{\alpha_{0}}{2^{k+1}}$ (if $\alpha$ is null, . $\alpha$ will be taken to be zero). If $\alpha$ and $\beta$ are strings, " $\alpha \beta$ " will denote the string consisting of $\alpha$ and $\beta$ in the order indicated; so, for example, " $0.010 \alpha$ " will denote $\frac{1}{4}+\frac{1}{8}(. \alpha)$, and if $\alpha=1101$, this will be the binary number 0.0101101 , or $45 / 128$.

If $A$ is any real number, " $[A]$ " will denote the greatest integer not greater than $A$, and " $\{A\}$ " will denote $A-[A]$. The distance from $A$ to the nearest integer, which equals the lesser of $\{A\}$ and $1-\{A\}$, will be denoted " $<A>$."

Let us now fix an integer $N>1$, and set $M=\left[\log _{2} N\right]$. For any $x \epsilon[0,1]$, let.$a_{0} a_{1} a_{2} \ldots$ be the nonterminating binary representation of $x$. Let $A=A(x)$ be the string $a_{M} a_{M-1} \ldots a_{1} a_{0}$. We 
will first consider the sequence $\mathscr{S}=x_{0}, x_{1}, \ldots$, with $x_{n}=\varphi_{2}(n)$. To estimate $S_{n}(x)$ we proceed as follows:

For $x_{n}$ to be less than $x$, exactly one of the following $M+2$ conditions must hold:

$$
\begin{aligned}
& \left(0^{\prime} .\right) \quad a_{0}>n_{0} \\
& \left(1^{\prime} .\right) \quad a_{0}=n_{0} ; a_{1}>n_{1} \\
& . \\
& . \\
& . \\
& \left(M^{\prime} .\right) \quad a_{0}=n_{0}, a_{1}=n_{1}, \ldots, a_{M-1}=n_{M-1} ; a_{M}>n_{M} \\
& \left(M+1^{\prime} .\right) \quad a_{0}=n_{0}, \ldots ., a_{M-1}=n_{M-1}, a_{M}=n_{M} .
\end{aligned}
$$

These are equivalent, respectively, to the following conditions:

$$
\begin{aligned}
& \text { (0.) } n \equiv 0(\bmod 2), a_{0}=1 \\
& \text { (1.) } n \equiv a_{0}\left(\bmod 2^{2}\right), a_{1}=1 \\
& \text { (2.) } n \equiv a_{0}+2 a_{1}\left(\bmod 2^{3}\right), a_{2}=1 \\
& \cdot . \\
& \text {. } \\
& \text { (M.) } n \equiv a_{0}+2 a_{1}+\ldots+2^{M-1} a_{M-1}\left(\bmod 2^{M+1}\right), a_{M}=1 \\
& (M+1 .) \quad n \equiv a_{0}+2 a_{1}+\ldots+2^{M} a_{M}\left(\bmod 2^{m+1}\right) .
\end{aligned}
$$

The number of integers $0 \leqslant n<N$ satisfying condition $(0$.) is

$$
a_{0}\left(\left[\frac{N}{2}\right]+1\right)
$$

the number satisfying condition (1.) is

$$
a_{1}\left(\left[\frac{N-a_{0}}{2^{2}}\right]+1\right)
$$

the number satisfying condition (2.) is

$$
a_{2}\left(\left[\frac{N-a_{0}-2 a_{1}}{2^{3}}\right]+1\right)
$$

etc. At most one $n$ satisfies condition $M+1$. Thus

$$
\sum_{i=0}^{M} a_{i}\left(\left[\frac{N-\left(a_{0}+2 a_{1}+\ldots .+2^{i-1} \dot{a}_{i-1}\right)}{2^{i+1}}\right]+1\right)
$$

differs from $S_{N}(x)$ by at most 1 . 
Now

$$
N x=N\left(\frac{a_{0}}{2}+\frac{a_{1}}{2^{2}}+\ldots\right)=\sum_{i=0}^{M} a_{i} \frac{N}{2^{i+1}}+\sum_{i=M+1}^{\infty} a_{i} \frac{N}{2^{i+1}} .
$$

By the definition of $M$ the second sum is between zero and 1 . If we now set

$$
d=d(x, N)=\sum_{i=0}^{M} a_{i}\left(\left[\frac{N-\left(a_{0}+\ldots+2^{i-1} a_{i-1}\right)}{2^{i+1}}\right]+1-\frac{N}{2^{i+1}}\right)
$$

then $d$ differs from $S_{N}(x)-N x$ by at most 2 ; and so in proving our Theorems, for which a difference which is $0(1)$ has no effect, we may deal with $d$ instead of $S_{N}(x)-N x$ throughout.

Now

$$
\frac{a_{0}+2 a_{1}+\ldots .+2^{i-1} a_{i-1}}{2^{i+1}}=.0 a_{i-1} a_{i-2} \ldots a_{0}
$$

(in binary notation), and may be written ".0 $0 A^{(i-1)}$ "; it is equal to $\frac{1}{2} \varphi_{2}\left(\left[2^{i} x\right]\right) .\left\{\frac{N}{2^{i+1}}\right\}$ may also be written “. $N^{(i)} . "$ Since

$$
\left[\frac{N-\left(a_{0}+. .+2^{i-1} a_{i-1}\right)}{2^{i+1}}\right]+1-\frac{N}{2^{i+1}}=\left[\left[\frac{N}{2^{i+1}}\right]+\left(. N^{(i)}-.0 A^{(i-1)}\right)\right]-\left[\frac{N}{2^{i+1}}\right]+1-. N^{(i)},
$$

we may write

$$
d=\sum_{i=0}^{M} a_{i} b_{i}, \text { where } b_{i}=\left\{\begin{array}{r}
1-. N^{(i)} \text { if } . N^{(i)} \geqslant .0 A^{(i-1)} \\
-. N^{(i)} \text { if } . N^{(i)}<.0 A^{(i-1)} .
\end{array}\right.
$$

We will first obtain a lower bound for $d: b_{i}<0$ only if $N_{i}=0$; therefore

$$
d \geqslant \sum_{\substack{N_{i}=0 \\ 0 \leqslant i \leqslant M}} a_{i} b_{i} \geqslant-\sum_{\substack{N_{i}=0 \\ 0 \leqslant i \leqslant M}} b_{i} \geqslant-\sum_{\substack{N_{i}=0 \\ 0 \leqslant i \leqslant M}} . N^{(i)} .
$$

To estimate this last sum, we first note that if the string $N$ (read from right to left) starts with a block of consecutive zeros, the $N^{(i)}$ corresponding to those digits are zero; and so that block of zeros may be removed from $N$. Similarly a block of ones occurring at the leftmost end of $N$ may be removed. What remains of $N$ can then be broken into disjoint substrings of the forms 01,001 , $0001, \ldots$ and $1,11,111, \ldots$ A substring 01 contributes one term to the sum.$- N^{(i)}$ for that $i$ which is the index of the zero in the substring. Since that.$N^{(i)}$ is less than $1 / 2$, the contribution of such a substring to the sum is less than $\mathbf{1} \mathbf{4}$ the length of the substring. Similarly each block of the form 001,0001, . . . contributes to the sum a set of terms whose total is less than $1 / 4$ the length of the block. Substrings of the form $1,11,111, \ldots$ contribute nothing to the sum. It follows that

$$
d>-\frac{M+1}{4}
$$

To obtain a sharp upper bound on $d$ we shall require some lemmas:

LEMMA 1. For a fixed integer $\mathrm{M} \geqslant 1$ and real number $\mathrm{x} \epsilon[0,1]$, and $\mathrm{A}=\mathrm{A}(\mathrm{x})$ as defined above, the maximum of $\mathrm{d}(\mathrm{x}, \mathrm{N})$ over all $\mathrm{N}$ between 0 and $2^{\mathrm{M}+1}-1$, is attained at $\mathrm{N}=2 \mathrm{~A}-2^{\mathrm{M}+1} \mathrm{a}_{\mathrm{M}}$. (i.e., $\mathrm{N}_{0}=0, \mathrm{~N}_{\mathrm{i}}=\mathrm{a}_{\mathrm{i}-1}$ for $\mathrm{i}=1,2, \ldots$. . .) 
Proof: $d(x, N)=\sum_{i=0}^{M} a_{i}(x) b_{i}(x, N)$. Changing any digit $N_{i}$ of $N$ changes $b_{j}$ only for $j \geqslant i$. If a change in some $N_{i}$ does not change the sign of some $b_{j}, j \geqslant i$, then it changes that $b_{j}$ by just $\frac{1}{2^{j-i+1}}$. For any $i$ between 0 and $M$, let $j_{0}$ be the 1 'st $j \geqslant i$ such that $a_{j}=1$; it then follows that if changing $N_{i}$ does not change the sign of $b_{j}$ for any $j \geqslant 1$, the change in $a_{j_{0}} b_{j_{0}}$ dominates the sum of all the changes in $d$ resulting from the change in $N_{i}$. On the other hand, no $b_{i}$ with $j \geqslant i$ changes sign unless $b_{j_{0}}$ does; so if the change in $N_{i}$ does change the sign of some $b_{j}$, the change in $a_{j_{0}} b_{j_{0}}$ is still dominating. It follows that the $N$ that maximizes $d$ must also maximize $\sum_{i=0}^{K} a_{i} b_{i}$ for every $K$ between zero and $M$.

Now let $a_{r_{1}}, a_{r_{2}} \ldots\left(r_{i}<r_{2}<\ldots\right)$, be the l's in $A$. To maximize $\sum_{i=0}^{r_{1}} a_{i} b_{i}$ we must clearly set $N_{0}=N_{1}=\ldots .=N_{r_{1}}=0$. Proceeding by induction, we assume that $N_{i}=a_{i-1}$ for $i=1,2, \ldots, r_{k}$, and try to define $N_{r_{k}+1}, \ldots, N_{r_{k+1}}$ so as to maximize $\sum_{i=0}^{r_{k+1}} a_{i} b_{i}$. If $r_{k+1}=r_{k}+1$, we must set $N_{r_{k}+1}$ $=1$, for otherwise $b_{r_{k}+1}$ would be negative. If $r_{k+1}>r_{k}+1$, it is clear that $b_{r_{k+1}}$ is maximized by setting $N_{r_{k}+1}=1, N_{r_{k}+2}=N_{r_{k}+3}=\ldots=N_{r_{k+1}}=0$. In either case $N_{i}=a_{i-1}$ for $i=r_{k}+1, \ldots$, $r_{k+1}$, proving the lemma.

Let us now, for any finite string $\alpha$ of zeros and ones, let $\delta(\alpha)$ be the maximum of $d(x, N)$ for $0 \leqslant N<2^{\|\alpha\|+1}$ and $A(x)=\alpha$. It remains to determine the maximum of $\delta(\alpha)$ over all strings $\alpha$ of a given length.

For any integer $L \geqslant 1$, let $\sigma_{L}$ and $\sigma_{L}^{\prime}$ be the strings $10101 \ldots 01$ and $0101 \ldots 01011$, respectively, each of length $2 L+1\left(\sigma_{1}=101, \sigma_{1}^{\prime}=011\right)$.

LEMma 2. If $0 \leqslant \mathrm{t} \leqslant 1$, the maximum of $\delta(\alpha)-\mathrm{t}(. \alpha)$ over all stringsi $\alpha$ of length $2 \mathrm{~L}+1$ occurs at $\alpha=\sigma_{\mathrm{L}}$; if $1 \leqslant \mathrm{t} \leqslant 2$ the maximum occurs at $\alpha=\sigma_{\mathrm{L}}^{\prime}$.

Proof: We first note that $\delta\left(\sigma_{L}^{\prime}\right)=\frac{2}{3} L+\frac{7}{9}+\frac{2}{9 \cdot 4^{L}}$; and that $. \sigma_{L}=\frac{2}{3}-\frac{1}{6 \cdot 4^{L}}$ and $. \sigma_{L}^{\prime}=\frac{1}{3}+\frac{1}{6 \cdot 4^{L}}$. The proof proceeds by induction on $L$. For $L=1$ the lemma is shown true by direct calculation. Assuming it true for $L$, if $\alpha$ is a string of length $2(L+1)+1$, then $\alpha=00 \beta$ or $01 \beta$ or $10 \beta$ or $11 \beta$, where $\|\beta\|=2 L+1$. In these 4 cases respectively we have:

$$
\begin{aligned}
\delta(\alpha)-t(. \alpha)= & \delta(\beta)-\frac{t}{4}(. \beta) \\
& \delta(\beta)-\left(1+\frac{t}{4}\right)(. \beta)+1-t / 4 \\
& \delta(\beta)-\left(\frac{1}{2}+\frac{t}{4}\right)(. \beta)+1-t / 2 \\
& \delta(\beta)-\left(\frac{3}{2}+\frac{t}{4}\right)(. \beta)+\frac{3}{2}-\frac{3 t}{4} ;
\end{aligned}
$$

we shall call these quantities $\delta_{1}, \delta_{2}, \delta_{3}$, and $\delta_{4}$. By the induction hypothesis, for $0 \leqslant t \leqslant 2, \delta_{1}$ and $\delta_{3}$ are maximized by taking $\beta=\sigma_{L}$, and $\delta_{2}$ and $\delta_{4}$ are maximized by $\beta=\sigma_{L}^{\prime}$. Direct calculation then shows that $\delta_{3}$ is the largest of the four when $0 \leqslant t \leqslant 1$, and $\delta_{2}$ is largest when $1 \leqslant t \leqslant 2$, which proves the lemma.

Thus in particular the maximum of $\delta(\alpha)$ over all strings $\alpha$ of length $2 L+1$ is $\delta\left(\sigma_{L}\right)$, which is equal to $\frac{1}{3}\|\alpha\|+0(1)$.

Furthermore, if $\alpha$ is a string of even length then $\alpha=0 \beta$ or $1 \beta$, where $\|\beta\|=2 L+1$ for some integer $L$. In the first case $\delta(\alpha)=\delta(\beta)$, and in the second $\delta(\alpha)=1-. \beta+\delta(\beta)$. Since $\beta<1, \alpha=1 \beta$ 
yields the larger $\delta$, and then, by Lemma 2., $\delta(\alpha)$ is maximal when $\beta=\sigma_{L}$ or $\sigma_{L}^{\prime}$; and once again the maximum of $\delta(\alpha)$ is $\frac{1}{3}\|\alpha\|+0(1)$.

Lemmas 1 and 2 together imply that for any positive integer $M$ the maximum of $d(x, N)$ for $0 \leqslant x \leqslant 1$ and $0 \leqslant N<2^{M+1}$ is $\frac{M+1}{3}+0(1)$, and Theorem 1. follows.

To prove Theorem 2., we shall first show:

Lemma 3. $\quad\left(\int_{0}^{1}\left(\mathrm{~S}_{\mathrm{N}}(\mathrm{x})-\mathrm{N} \mathrm{x}\right)^{2} \mathrm{dx}\right)^{1 / 2}=\frac{1}{2} \sum_{\mathrm{i}=1}^{\infty}\left\langle\frac{\mathrm{N}}{2^{\mathrm{i}}}\right\rangle+0(1)$.

Proof: First of all, it is sufficient to prove the equation with $d(x, N)$ in place of $S_{N}(x)-N x$. For fixed $N$, and $i=0,1, \ldots, M$ let $f_{i}(x)=a_{i}(x) b_{i}(x, N)$. Then $d(x, N)=f_{0}(x)+f_{1}(x)+\ldots+f_{M}(x)$, and

$$
\int_{0}^{1} d^{2}(x, N) d x=\left(\sum_{i=0}^{M} \int_{0}^{1} f_{i}\right)^{2}+\sum_{i=0}^{M}\left(\int_{0}^{1} f_{i}^{2}-\left(\int_{0}^{1} f_{i}\right)^{2}\right)+2 \sum_{0 \leqslant i<j \leqslant M}\left(\int_{0}^{1} f_{i} f_{j}-\int_{0}^{1} f_{i} \int_{0}^{1} f_{j}\right) .
$$

To evaluate the first term on the right of (16), we first define $C_{i}$, for each $i$, by

$$
C_{i}(x)=\left\{\begin{array}{l}
1-. N^{(i)} \text { if } . N^{(i)} \geqslant .0 A^{(i+1)}+\frac{1}{2^{i+1}} \\
-. N^{(i)} \text { otherwise }
\end{array}\right.
$$

and note that $C_{i}=b_{i}$ unless, $0 A^{(i-1)} \leqslant . N^{(i)}<.0 A^{(i-1)}+\frac{1}{2^{i+1}}$, when $C_{i}=b_{i}-1$. Now $.0 A^{(i-1)}=$ $\frac{1}{2} \varphi_{2}\left(\left[2^{i} x\right]\right)$ and so is constant on each interval $\left(\frac{r}{2^{i}}, \frac{r+1}{2^{i}}\right), r=0,1, \ldots, 2^{i}-1$; and its values on different intervals differ by at least $2^{-i}$. Thus $C_{i}$ and $b_{i}$ are equal everywhere except perhaps on one interval of length $2^{-i}$, so that if we set $F_{i}(x)=a_{i}(x) C_{i}(x)$, we have

$$
\int_{0}^{1}\left|F_{i}(x)-f_{i}(x)\right| d x \leqslant \frac{1}{2^{i}} \text {, and }\left|\sum_{i=0}^{M} \int^{1} F_{i}-\sum_{i=0}^{M} \int_{0}^{1} f_{i}\right| \leqslant 2 .
$$

Since $a_{i}$ is zero on one half of each interval $\left(\frac{r}{2^{i}}, \frac{r+1}{2^{i}}\right)$ and one on the other half, while $C_{i}$ is constant on the interval, $\int_{0}^{1} F_{i}(x) d x=\frac{1}{2} \int_{0}^{1} C_{1}(x) d x$. The values $.0 A^{(i-1)}+\frac{1}{2^{i+1}}$ takes on these $2^{i}$ intervals are just the numbers $\frac{1}{2^{i+1}}, \frac{2}{2^{i+1}}, \ldots, \frac{2^{i}}{2^{i+1}}$. So if $. N^{(i)} \geqslant \frac{1}{2}, C_{i}=1-. N^{(i)}$ throughout $(0,1)$ and $\int_{0}^{1} F_{i}=$ $\frac{1}{2}\left(1-. N^{(i)}\right)=\frac{1}{2}\left\langle\frac{N}{2^{i+1}}\right\rangle \cdot$ If $. N^{(i)}<\frac{1}{2}$, we may write. $N^{(i)}=K / 2^{i+1}$, where $K$ is an integer between 0 and $2^{i}-1$. It's then easy to see that $. N^{(i)}<.0 A^{(i-1)}+\frac{1}{2^{i+1}}$ on just $2^{i}-K$ of the above intervals. Then $C_{i}(x)=. N^{(i)}$ on a set of measure $1-K / 2^{i}$ and $1-. N^{(i)}$ on a set of measure $K / 2^{i}$; so that $\int_{0}^{1} F_{i}=\frac{. N^{(i)}}{2}$ $=\frac{1}{2}\left\langle\frac{N}{2^{i+1}}\right\rangle$ once again. Therefore, by (18),

$$
\sum_{i=0}^{M} \int_{0}^{1} f_{i}=\frac{1}{2} \sum_{i=0}^{M}\left\langle\frac{N}{2^{i+1}}\right\rangle+0(1)=\frac{1}{2} \sum_{i=1}^{\infty}\left\langle\frac{N}{2^{i}}\right\rangle+0(1) .
$$

Lemma 3. will now follow if we show that the second and third terms on the right in (16) are 
each $0(\log N)$. For the second term this is obvious, since $\left|f_{i}(x)\right| \leqslant 1$. To bound the $3 \mathrm{~d}$ term we proceed as follows:

For $0 \leqslant i \leqslant M$, set $b_{i}(x)=-. N^{(i)}+e_{i}(x)$, and $y_{i}(x)=.0 A^{(i-1)}$.

Then

$$
e_{i}=\left\{\begin{array}{l}
1 \text { if } . N^{(i)} \geqslant y_{i} \\
0 \text { if } . N^{i}<y_{i}
\end{array},\right.
$$

and

$$
\int_{0}^{1} f_{i} f_{i}-\int_{0}^{1} f_{i} \int_{0}^{1} f_{i}=\frac{1}{4}\left(\int_{0}^{1} e_{i} e_{i}-\int_{0}^{1} e_{i} \int_{0}^{1} e_{i}\right)
$$

Now for any $i$ and $j, i<j$, set $z_{i, j}(x)=y_{j}(x)-2^{i-j} y_{i}(x)$. Recalling that $y_{i}=1 / 2 \varphi_{2}\left(\left[2^{i} x\right]\right)$ and noting that $\varphi_{2}\left(\left[2^{i} x\right]\right)=\left\{2^{j-i} \varphi_{2}\left(\left[2^{j} x\right]\right)\right\}$, we see that $z_{j, i}$ takes on, in each interval $\left(\frac{r}{2^{i}}, \frac{r+1}{2^{i}}\right)$, the $2^{j-i}$ values $\frac{l}{2^{j-i+1}}, l=0,1, \ldots ., 2^{j-i}-1$; and each value is taken on an interval of length $2^{-j}$. Therefore $z_{j, i}$, and $y_{i}$ are independent functions of $x$, and if we define $e_{j}^{*}(x)=e_{j, i}^{*}(x)$ by

$$
e_{j}^{*}=\left\{\begin{array}{l}
1 \text { if } . N^{(i)} \geqslant z_{j} \\
0 \text { if } . N^{(i)}<z_{j}
\end{array},\right.
$$

we can conclude that

$$
\int_{0}^{1} e_{i} e_{j}^{*}-\int_{0}^{1} e_{i} \int_{0}^{1} e_{j}^{*}=0
$$

By reasoning as we did above about $b_{i}$ and $C_{i}$ we can see that $e_{i}(x)$ and $e_{j}^{*}(x)$ are equal except on a set of measure at most $2^{i-j+1}$, and there $\left|e_{j}^{*}(x)-e_{j}(x)\right|=1$. Therefore, by (23)

$$
\left|\int_{0}^{1} e_{i} e_{j}-\int_{0}^{1} e_{i} \int_{0}^{1} e_{j}\right|=\left|\int_{0}^{1} e_{i}\left(e_{j}-e_{j}^{*}\right)-\int_{0}^{1} e_{i} \int_{0}^{1}\left(e_{j}-e_{j}^{*}\right)\right| \leqslant 2 \int_{0}^{1}\left|e_{j}-e_{j}^{*}\right| \leqslant \frac{4}{2^{j-i}} .
$$

From (21), therefore,

$$
\sum_{0 \leqslant i<j \leqslant M}\left(\int_{0}^{1} f_{i} f_{j}-\int_{0}^{1} f_{i} \int_{0}^{1} f_{j}\right) \leqslant \sum_{0 \leqslant i<j \leqslant M} \frac{1}{2^{i-j}}<M,
$$

which completes the proof of the lemma.

To prove Theorem 2 we must now show that

$$
\sum_{i=0}^{M}\left\langle\frac{N}{2^{i+1}}\right\rangle \leqslant \frac{1}{3} \log _{2} N+0(1)
$$

and that the constant $1 / 3$ in (24) is best possible. We have seen that $\sum_{i=0}^{M}\left\langle\frac{N}{2^{i+1}}\right\rangle=\sum_{i=0}^{M} \min \left(. N^{(i)}\right.$, $1-. N^{(i)}$ ); and we shall show that the last sum is maximized (over all $0 \leqslant N<2^{M+1}$ ) when $N$ is of the form . . . 010101:

For $M=0$, this is obvious. If $M=K$, then $N=0 N^{\prime}$ or $1 N^{\prime}$, where $N^{\prime}$ is a string with $M=K-1$. In the first case the sum is $\frac{1}{2}\left(. N^{\prime}\right)+\sum_{i=0}^{K-1} \min \left(. N^{(i)}, 1-. N^{(i)}\right)$, and in the second it is $\frac{1}{2}\left(1-. N^{\prime}\right)+\sum_{i=0}^{K-1}$ 
$\min \left(. N^{(i)}, 1-. N^{(i)}\right)$. The first is larger if the leftmost digit of $N^{\prime}$ is 1 , while the second is larger if the leftmost digit of $N^{\prime}$ is zero; which completes the induction. For $N$ of the form specified, $\sum_{i=0}^{M}\left\langle\frac{N}{2^{i+1}}\right\rangle$ is equal to $1 / 3 \log _{2} N+0(1)$, so (24) is established, together with the fact that the constant $1 / 3$ cannot be improved upon.

To derive Theorems 3 and 4 from Theorems 1 and 2 we will use a device of K. F. Roth [3]. For any $N$, and $0 \leqslant x \leqslant 1$ and $0 \leqslant y \leqslant 1$, let $n=n(y)=[N y]$. Then $S_{N}(x, y)$ for the sequence $\mathscr{S}^{\text {' }}$ is equal to $S_{n}(x)$ for the sequence $\mathscr{S}$. Furthermore $|N x y-n x| \leqslant 1$, so that

$$
\sup _{0 \leqslant x, y \leqslant 1}\left|S_{N}(x, y)-N x y\right| \text { differs from } \sup _{\substack{0 \leqslant x \leqslant 1 \\ 0<n<N}}\left|S_{n}(x)-n x\right| \text { by no more }
$$

than 1. From Theorem 1,

$$
\left|S_{n}(x)-n x\right| \leqslant \frac{1}{3} \log _{2} n+0(1) \leqslant \frac{1}{3} \log _{2} N+0(1)
$$

and from the proof of Theorem 1 , if $n$ is the largest integer between 0 and $N$ whose binary representation is of the form $10101 \ldots 01$,

$$
\sup \left|S_{n}(x)-n x\right|=\frac{1}{3}\|n\|+0(1)=\frac{1}{3}\|N\|+0(1)
$$

Theorem 3 follows.

To prove Theorem 4. we use the device of Roth to replace

$$
\left(\int_{0}^{1} \int_{0}^{1}\left(S_{N}(x, y)-N x y\right)^{2} d y d y\right)^{1 / 2} \text { by }\left(\int_{0}^{1} \int_{0}^{1}\left(S_{n(y)}(x)-x n(y)\right)^{2} d x d y\right)^{1 / 2}
$$

the latter, in turn, differs by only $0(1)$ from

$$
\left(\int_{0}^{1}\left(\frac{1}{2} \sum_{i=0}^{M}\left\langle\frac{n(y)}{2^{i+1}}\right\rangle\right)^{2} d y\right)^{1 / 2}
$$

Setting $g_{i}(y)=\left\langle\frac{n(y)}{2^{i+1}}\right\rangle$, we can write

$\int_{0}^{1}\left(\sum_{i=0}^{M}\left\langle\frac{n}{2^{i+1}}\right\rangle\right)^{2} d y=\left(\sum_{i=0}^{M} \int_{0}^{1} g_{i}\right)^{2}+\sum_{i=0}^{M}\left(\int_{0}^{1} g_{i}^{2}-\left(\int_{0}^{1} g_{i}\right)^{2}\right)+2 \sum_{0 \leqslant i<j \leqslant M}\left(\int_{0}^{1} g_{i} g_{j}-\int_{0}^{1} g_{i} \int_{0}^{1} g_{j}\right)$.

As in the analysis of (16), we wish to show that the second and third terms on the right of (26) are each $0(M)$. For the second this is obvious; for the third we proceed so: For each $i$ and $j>i$, set $g_{j, i}(y)=\left\langle\left\{\frac{n}{2^{j}}\right\}-2^{i-j}\left\{\frac{n}{2^{i}}\right\}\right\rangle ; g_{j, i}$ is then a function of only the $M-j$ th through $M-i+1$ th digits of the binary representation of $y$, and so is independent of $g_{i}$, which is a function of the $M-i$ th through $M$ th digits. Furthermore $\int_{0}^{1}\left|g_{j, i}(x)-g_{j}(x)\right| d x \leqslant \frac{2}{2^{j-i}}$; that the third term is $0(M)$ follows as in the proof of Lemma 3.

To evaluate the first term on the right of $(26)$, we note that

$$
\int_{0}^{1} g_{i}(y) d y=\int_{0}^{1} \frac{[N y]}{2^{i+1}} d y=\frac{1}{N} \sum_{r=0}^{N-1}\left\langle\frac{r}{2^{i+1}}\right\rangle
$$


Since $\langle x\rangle=\langle\{x\}\rangle$, the sum in (27) can be written as

where

$$
\left[\frac{N}{2^{i+1}}\right] \sum_{r=0}^{2^{i+1}-1}\left\langle\frac{r}{2^{i+1}}\right\rangle+E_{i}=\frac{2^{i+1}}{4}\left[\frac{N}{2^{i+1}}\right]+E_{i},
$$

$$
0 \leqslant E_{i}=\sum_{r=2^{i+1}\left[\frac{N}{2^{i+1}}\right]}^{N-1}\left\langle\frac{r}{2^{i+1}}\right\rangle<\frac{2^{i+1}}{4}
$$

Therefore

$$
\begin{aligned}
N \int_{0}^{1} g_{i} & =\frac{2^{i+1}}{4}\left(\frac{N}{2^{i+1}}-\left\{\frac{N}{2^{i+1}}\right\}+\frac{4}{2^{i+1}} E_{i}\right) \\
& =\frac{N}{4}+\frac{2^{i+1}}{4} \theta_{i},
\end{aligned}
$$

where $\left|\theta_{i}\right| \nless 1$.

So

$$
\sum_{i=0}^{M} \int_{0}^{1} g_{i}=\frac{M+1}{4}+\frac{\theta}{N} \sum_{i=0}^{M} 2^{i-1}
$$

where $|\theta|<1$. Since $\sum_{i=0}^{M} 2^{i-1}<2^{M} \leqslant N$, we can conclude that

$$
\sum_{i=0}^{M} \int_{0}^{1} g_{i}=\frac{M}{4}+0(1)
$$

and Theorem 4. follows.

4. An attempt to analyze the higher-dimensional sequences of Hammersley and Halton along the above lines was unsuccessful, due to the greater complication of those sequences. Analogs, for those sequences, of Theorems 1 and 3 would be very useful in application of the sequences to multiple numerical quadrature.

Intuitively, van der Corput's sequence $\mathscr{S}$ seems to be about as evenly distributed as possible. This feeling, together with Theorem 1 , leads us to the conjecture in section 1 that K. F. Roth's lower bound on $D_{N}^{*}$ can be improved.

\section{References}

[1] J. W. S. Cassels, An Introduction to Diophantine Approximation (Cambridge University Press, 1957).

[2] E. Hlawka, Funktionen von beschrankter Variation in der Theorie der Gleichverteilung. Annali di Matematica (IV) $54,1961$.

[3] K. F. Roth, On irregularities of distribution, Mathematika 1 (1954).

[4] J. G. van der Corput, Verteilungsfunktionen, Proc. Kon. Ned. Akad. v. Wetensch., Section of Sciences 38 (1935).

[5] T. van Aardenne-Ehrenfest, Proof of the impossibility of a just distribution of an infinite sequence of points over an interval, Proc. Kon. Ned. Akad. v. Wetensch. 48 (1945).

[6] J. M. Hammersley, Monte Carlo methods for solving multivariable problems, Annals of the New York Academy of Sciences, 86 (1960).

[7] J. H. Halton, On the efficiency of certain quasi-random sequences of points in evaluating multi-dimensional integrals. Numerische Math. 2, 1960.

(Paper 70B2-172) 